\title{
Mapping Solvation Heterogeneity in Live Cells by Hyperspectral Stimulated Raman Scattering
} Microscopy

Xiaoqi Lang ${ }^{1}$ and Kevin Welsher ${ }^{1 *}$

${ }^{1}$ Department of Chemistry, Duke University

\begin{abstract}
:
Water provides a dynamic matrix in which all biochemical processes occur in living organisms. The structure and dynamics of intracellular water constitute the cornerstone for understanding all aspects of cellular function. Fundamentally, direct visualization of subcellular solvation heterogeneity is essential but remains challenging with commonly used NMR methods due to poor spatial resolution. To explore this question, we demonstrate a vibrational-shift imaging approach by combining the spectral-focusing hyperspectral stimulated Raman scattering (hsSRS) technique with an environmentally-sensitive nitrile probe. The sensing ability of a near-infrared nitrile-containing molecule is validated in the solution phase, microscopic droplets and cellular environments. Finally, we quantitatively measure the subcellular solvation variance between the cytoplasm (29.5\%, S.E. $1.8 \%)$ and the nucleus (57.3\%, S.E. 1.0\%), which is in good agreement with previous studies. This work sheds light on heterogenous solvation in live systems using coherent Raman microscopy and opens up new avenues to explore environmental variance in complex systems with high spatiotemporal resolution.
\end{abstract}

\section{Introduction}

Water, as the most abundant molecule in cells, is not merely "life's solvent", but an active participant in a wide range of biochemical and biophysical processes, such as protein folding, enzyme catalysis, electron and proton transfer, and molecular recognition..$^{1-3}$ However, the extent to which intracellular water differs from the familiar "bulk" water remains unclear. ${ }^{2-5}$ The physiochemical state and structure of water in the cell has an enormous impact on how well studies of biochemical processes in dilute solution reflect on the biochemistry of the cell. A number of different methods have been applied in order to tease out the structure and dynamics of water in the cytoplasm. The earliest and most frequently used method to probe water 
structure is nuclear magnetic resonance (NMR) spectroscopy. ${ }^{6,7}$ Pulsed NMR studies on a variety of sources (living frog muscles, ${ }^{8}$ brine shrimp, ${ }^{9}$ Xenopus laevis oocytes, ${ }^{10}$ and mitochondria ${ }^{11}$ ) have revealed decreased $\mathrm{T} 1$ and $\mathrm{T} 2$ proton relaxation times, indicative of restricted water mobility, similar to what is observed in engineered nanospaces. ${ }^{12}$ Dielectric relaxation spectroscopy, which measures the response to high-frequency EM fields, has also shown decreased water mobility in ocular tissue, ${ }^{13}$ plant leaves, ${ }^{14}$ and collagen solutions, ${ }^{15}$ consistent with the NMR studies. Quasi-elastic neutron scattering (QENS) studies have been less conclusive. The earliest studies seemed to agree with both the NMR and dielectric relaxation spectroscopy data, showing decreased cytosolic water dynamics in brine shrimp. ${ }^{16}$ Somewhat more recent studies have since concluded that there is only a small difference between cytosolic and bulk water. ${ }^{17,18}$ To complicate the situation further, a recent QENS study found that cytosolic water exhibits decreased diffusion compared to bulk water, with the surprising result that this difference from bulk water behavior became even more drastic with the addition of cisplatin. ${ }^{19}$

Despite the long history of studying the dynamics of cytosolic water, much remains unclear and unresolved. The NMR data seem to agree that cytosolic water shows reduced diffusion compared to bulk water. However, it is not clear what the spatial scale of this inhibited diffusion is. Is cytosolic water unstructured and sub-diffusive on the micron scale, or is it genuinely structured at the molecular scale? To our knowledge, there is only one report of NMR microscopy used to probe this issue, finding decreased mobility of cytoand nucleo-plasmic water relative to the bulk. ${ }^{10}$ The disagreement among QENS studies may be due to the fact that a dried cell pellet must be prepared, potentially complicating the analysis, particularly if the estimation of remaining extracellular water is inaccurate or if there are substantial differences in water dynamics between cells or sub-cellular compartments. It is clear that a new approach is needed which can probe water structure and solvation at the sub-cellular level. This will allow fundamental questions to be addressed, such as the spatial heterogeneity of solvation environments inside cells and subcellular compartments, and further, how closely they are related to biomolecular structure and dynamics. 
Vibrational-based techniques [particularly infrared absorption (IR) spectroscopy] have proven to be powerful tools to probe water dynamics ${ }^{20,21}$ and the microenvironments associated with biomolecules with high structural and temporal resolution. These studies are especially informative when coupled with vibrational probes, including carbon-deuterium bonds, ${ }^{22-24}$ azides, ${ }^{25}$ carbonyl ${ }^{26-28}$ and nitrile groups ${ }^{29-32}$ to name a few. These IR probes,${ }^{33}$ owing to the environmental sensitivity of molecular vibrations,${ }^{34}$ have been employed with success in gaining site-specific insights regarding the electrostatics, ${ }^{26,27,32,35}$ stability and folding, ${ }^{23,31}$ protonation $^{24}$, and dynamics ${ }^{36}$ of proteins. Among them, nitriles ${ }^{30}$ and thiocyanates ${ }^{35,37,38}$ are particularly appropriate reporters for intracellular solvation due to their chemical stability, relatively intense vibrational features in a low background spectral region, and their proclivity towards hydrogen bonding (H-bonding).

Previous studies ${ }^{37-42}$ have used nitriles to probe solvation and water accessibility of different sites within proteins. However, this sensitive tool has yet to be applied to investigate the distribution of solvation and H-bonding environments at the cellular scale. The reasons for this are manifold. First, the primary method used to study this effect has been IR absorption spectroscopy, which is not feasible for biological imaging due to long wavelengths, poor signal-to-noise inherent to an absorption measurement, and strong water interference. The alternative vibrational spectroscopy to IR absorption is spontaneous Raman scattering, which can be easily applied to microscopy owing to the Stokes shift between the incoming and scattered photons. However, extremely small Raman cross-sections ${ }^{43}$ limit the ability to sense very small (several wavenumbers) shifts at low probe concentrations. Furthermore, the low signal-to-noise ratio means a 2D Raman image of a biological sample could take up to an hour to complete. ${ }^{44}$ Although the use of paralleldetection techniques and the combination of resonance Raman effect have reduced the acquisition time to a few tens of minutes, ${ }^{45}$ current conditions are still not optimal to probe solvation dynamics in live cells. To rapidly characterize water structure inside cells, new microscopic techniques with high detection sensitivity and fast acquisition speed are needed. 
Stimulated Raman scattering (SRS) microscopy has emerged as a powerful technique for elucidating the complex structure and dynamics of biological systems by offering unprecedented imaging speed, high detection sensitivity, and high spatial resolution. ${ }^{46}$ In this paper, we demonstrate that the combination of SRS and a near-resonant probe can extend the sensing capability of nitriles to probe solvation and $\mathrm{H}-$ bonding characteristics in live cells with sub-micron resolution. Recent work by Min and coworkers ${ }^{47}$ have shown that a SRS-modulated fluorescence technique can be used to measure solvation heterogeneity in living cells. Here we demonstrate that a direct vibrational measurement can be similarly applied, potentially reducing the reliance on a fluorescent label and dramatically increasing the application scope of this novel approach.

\section{Experimental Methods}

The commonly demonstrated narrowband SRS modality is not amenable to measuring dynamic processes due to slow sequential wavelength-tuning required to acquire spectra using picosecond lasers. To rapidly retrieve spectroscopic information, we apply a spectral-focusing method ${ }^{48,49}$ to enable hyperspectral SRS (hsSRS) imaging. Figure 1(a) illustrates the instrumental set-up. Briefly, two synchronized broadband femtosecond lasers are linearly chirped by propagating through long H-ZF52A glass rods so that higher frequencies are delayed in time [Fig. 1(b)]. The fixed $1040 \mathrm{~nm}$ (Stokes) beam is modulated at $10 \mathrm{MHz}$ by an electro-optic modulator (EOM). The Stokes beam is spatially and temporally overlapped with a wavelength-tunable (pump) beam and focused on a diffraction-limited volume to provide SRS excitation. A lock-in amplifier is used to extract the stimulated Raman loss (SRL) signal from the unmodulated pump beam. The vibrational excitation is governed by the interference of the pump and the Stokes field; therefore, the spectral resolution is determined by the Fourier limit of their temporal envelope.$^{50}$ By chirping the pump and Stokes pulses equally in time, it is possible to drive a narrow vibrational frequency range, even though the individual pulses are spectrally broad. The linear chirp allows spectra to be attained by simply adjusting the arrival time of the Stokes beam using a mechanical delay stage (DLS). The DLS position was calibrated by comparing hsSRS spectra with spontaneous Raman spectra for several nitriles (Fig. S1). To construct 
an image from hsSRS data, the overlapped pump and Stokes beams are raster-scanned with a 2D galvo mirror across the sample. A hyperspectral image stack is then created by recording an image at different DLS positions, resulting in an $\mathrm{XY} \Omega$ (where $\Omega$ is frequency) data set where the vibrational Raman spectrum of each pixel can be retrieved and analyzed.

(a)

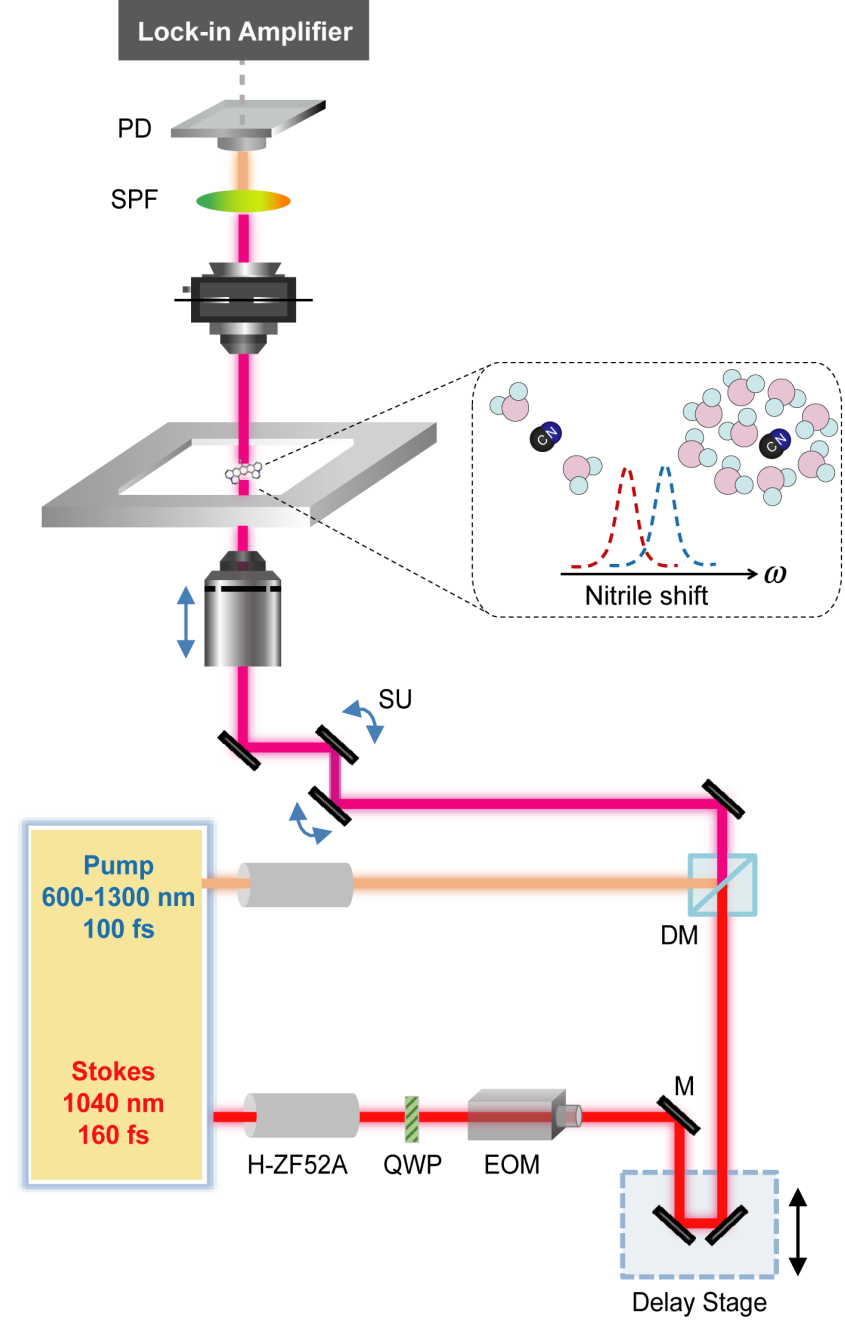

(b)
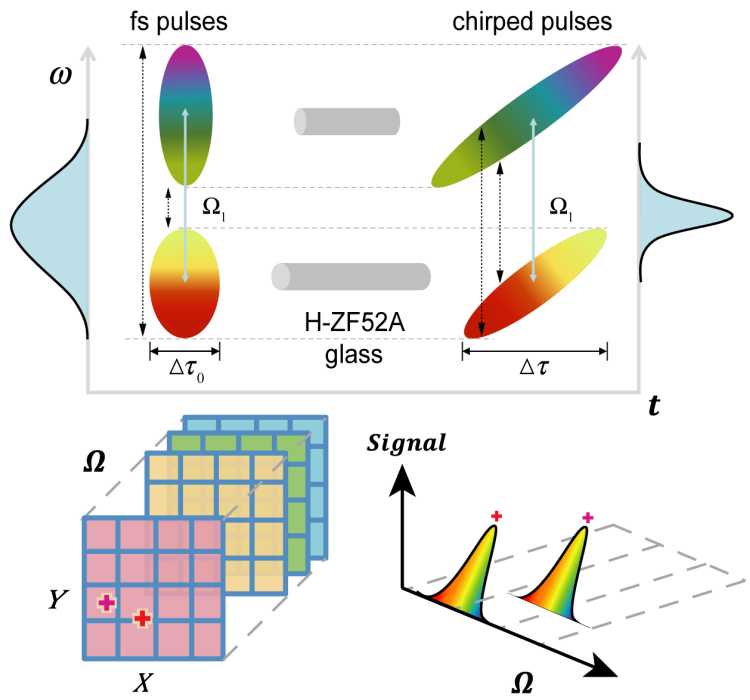

Wavelength $\lambda_{\text {ex }} \mathrm{nm}$

(c)

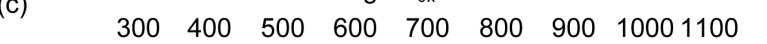

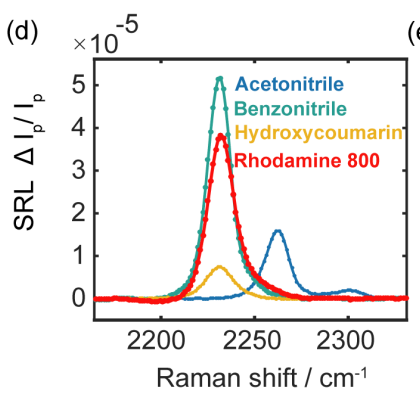

(e) $\times 10^{-5}$

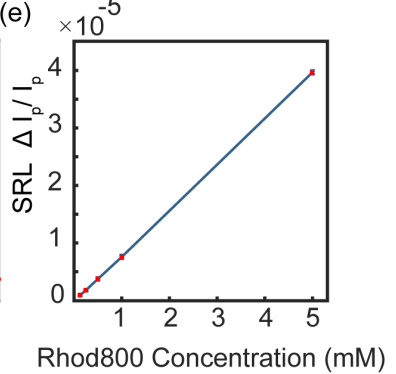

Figure 1. Hyperspectral SRS (hsSRS) Microscopy. (a) Schematic of hsSRS microscopy based on a dual-broadband laser system. H-ZF52A, glass rods used for pulse chirping; QWP, quarter-wave plate; EOM, electro-optical modulator; M, mirror; DM, dichroic mirror; SU, scanning unit; SPF, short-pass filter; PD, photodiode. (b) Upper: Principle of spectral-focusing method. Transform-limited fs laser pulses are temporally short but spectrally broad (depicted as elliptical shape in the frequency-time diagram), generating a broad instantaneous frequency difference (IFD). After passing through a dispersive medium (where lower frequencies emerge first, higher frequencies are delayed), the pulses stretch out in time while the spectral range of the pulse remains constant (tilted and stretched elliptical shape). The IFD between two equally chirped pulses is constant with time, but can be tuned by varying the overlap of the two 
pulses, thus allowing spectral tuning (Fig. S1). Bottom: SRS imaging and spectroscopic analysis based on hsSRS stack acquired by delay scanning. (c-d) Electronic pre-resonance stimulated Raman scattering (epr-SRS). The pump wavelength is set at $842 \mathrm{~nm}$. (c) Absorption profiles of four nitriles at different SRS regimes. Non-resonant $\left(\omega_{0} \gg\right.$ $\left.\omega_{\text {pump }}\right)$ : acetonitrile (blue line), benzonitrile (cyan line), 3-cyano-7-hydroxycoumarin (yellow line). Pre-resonant ( $\omega_{0}$ $\left.\omega_{\text {pump }} \approx 3 \Gamma, \Gamma \approx 700 \mathrm{~cm}^{-1}\right)$ : Rhod800 ( $\lambda_{\text {abs }} \approx 700 \mathrm{~nm}$, red line). (d) hsSRS spectra of acetonitrile ( $2 \mathrm{M}$, water, blue line), benzonitrile (1 M, DMSO, cyan line), 3-cyano-7-hydroxycoumarin (50 mM, DMSO, yellow line) and Rhod800 (1 $\mathrm{mM}$, DMSO, red line). (e) Linear dependence of epr-SRS signal on Rhod800 concentration (DMSO) with $3 \mathrm{~ms}$ time constant at $\mathrm{P}_{\text {pump }}=20 \mathrm{~mW}, \mathrm{P}_{\text {Stokes }}=26 \mathrm{~mW}$.

\section{Results and Discussion}

This section is broken into four parts. In part A, we demonstrate that a near-resonant vibrational probe can be used to report nitrile solvation in the solution phase. In part B, we translate this solvation sensing to the microscale to investigate the composition of micron-sized droplets. In part $\mathrm{C}$, we detail the application and results of this local solvation probe to live cells. In part D, we compare this study with previous work.

\section{A. Solution-phase sensing of water content with hsSRS and a near-resonant probe}

We demonstrate the sensitivity of the hsSRS spectra to different solvation environments by first performing solvatochromic experiments on a simple nitrile: acetonitrile (Fig. S2). Different water-acetonitrile mixtures were tested by recording the SRS signal at different positions of the delay stage, effectively probing different frequencies. To calibrate, the hsSRS spectra obtained with the delay stage were compared with the spontaneous Raman spectra measured using a commercial Raman spectrometer. In both cases, a monotonic blue-shift (to higher frequency) is observed with increasing water proportion in the mixture, showing the ability of hsSRS to sense differences in water content.

An ideal molecular sensor for probing solvation should offer a large Raman scattering cross-section to increase signal and minimize potential perturbations to the native environment. It has been reported that the SRS cross-section can be drastically enhanced by operating at the electronic pre-resonance (epr) condition. ${ }^{51}$ To take advantage of this mechanism, the near-IR dye Rhodamine 800 (Rhod800) was selected, which contains a conjugated nitrile bond and absorbs around $700 \mathrm{~nm}$ (close to the pump excitation at 842 nm). Due to electronic-vibrational coupling, the Raman cross-section of Rhod800 is estimated to be 200- 
fold higher than a non-resonant dye with a similar structure [3-cyano-7-hydroxycoumarin, Fig. 1(d)]. The linear concentration dependence of Rhod800 was then confirmed with a sensitivity down to $100 \mu \mathrm{M}$ [Fig. 1(e)]. We note here that other non-linear processes, such as cross-phase modulation (XPM), can lead to a non-uniform background in the hsSRS spectra. ${ }^{52,53}$ Fig. S3 shows an example of Rhod800 spectrum in DMSO, where a broad baseline accompanies the sharp nitrile Raman peak at $2228.5 \mathrm{~cm}^{-1}$. To account for these background effects, each raw SRS spectrum is corrected by subtracting the baseline (fit to a $6^{\text {th }}$ degree polynomial). The background-corrected spectrum is fit to a Lorentzian, the location of the peak maximum is extracted as the nitrile shift.

In aprotic environments, the observed frequency shift of nitriles is dictated by the vibrational Stark effect (VSE), where a red-shift represents an increase in solvent polarity [Fig. S4(b-c)], following a linear frequency versus field strength relationship. ${ }^{41}$ In protic solvents, however, a blue-shift is typically observed in IR spectra due to hydrogen bonding regardless of the solvent polarity [Fig. S4(a)]. In IR spectra, these two effects are conflated, though tandem ${ }^{13} \mathrm{C}$ NMR/IR measurements have been used to isolate and quantify the H-bonding contribution $\left(\sim 10 \mathrm{~cm}^{-1}\right.$ frequency shift) from the pure electrostatic effects of the local environment. ${ }^{37}$ To measure the response of Rhod800 to a range of solvation environment, we performed a hsSRS scans on Rhod800 (1 mM) dissolved in different DMSO-water mixtures. Since both nitriles and DMSO are H-bond acceptors, it is expected that nitriles will only form $\mathrm{H}$-bonds with the water molecules in these mixtures. Figure 2(a) shows an overall blue-shift (about $4.7 \mathrm{~cm}^{-1}$ ) with increasing water content (from $0 \%$ to $85 \%$ ), following a sigmoidal trend in $\omega_{\mathrm{CN}}$. This blue-shift is similar to previous solvatochromic results with benzonitrile [Fig. S4(a) $]^{41}$, implying the hydrogen bonding is the determining factor in Rhod 800 peak position. 

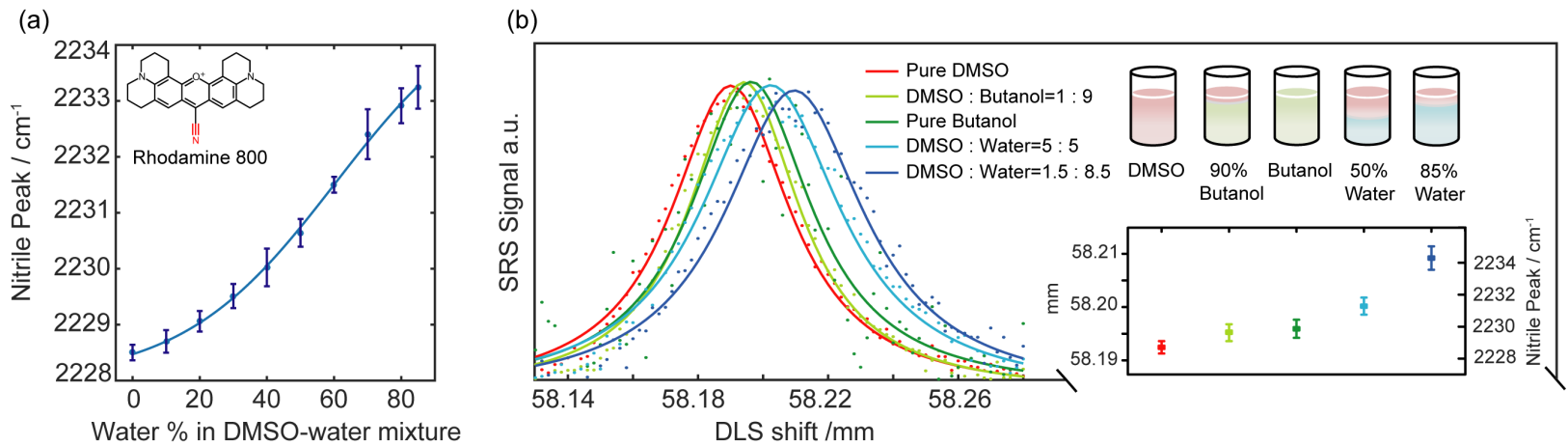

Figure 2. Solution-phase solvatochromic measurements for Rhodamine 800. (a) Nitrile shift of Rhod800 (1 mM) versus water content in DMSO-water binary mixtures as measured by hsSRS $\left(\tau=3 \mathrm{~ms}, \mathrm{P}_{\text {pump }}=20 \mathrm{~mW}\right.$, Pstokes $=26$ $\mathrm{mW}$ ). (b) Normalized hsSRS spectra of Rhod800 in different solvents $\left(\tau=30 \mathrm{~ms}, \mathrm{P}_{\text {pump }}=20 \mathrm{~mW}\right.$, Pstokes $\left.=26 \mathrm{~mW}\right)$. These set of samples were centrifuged to remove all possible aggregates before being measured on the scope. Dotted line: background-corrected raw data; solid line: Lorentzian fitting curve. Inset box: plot of nitrile shifts in different solvents. Over 10 scans were obtained for each sample.

\section{B. Probing microscopic heterogeneities in H-bonding with hsSRS}

To validate the feasibility of this nitrile-shift strategy in resolving microscopic differences in the local solvation environment, we demonstrate hsSRS imaging of Rhod800 distributed in butanol-water emulsions containing micron-scale sized droplets. In the solution-phase, the nitrile peak positions differ by about 5 $\mathrm{cm}^{-1}$ between butanol- and water-rich solutions [Fig. 2(b)]. An emulsion was created by vortexing together 90\% butanol/DMSO and 85\% water/DMSO mixtures, each containing $1 \mathrm{mM}$ Rhod800, at a ratio of 1:5 to generate butanol droplets $(<5 \mu \mathrm{m})$ within water-rich surroundings [Fig. 3(a)]. The hsSRS spectra of these droplets are dominated by $\mathrm{CH}_{2}$ stretching features in the high wavenumber region (2800 to $\left.3000 \mathrm{~cm}^{-1}\right)$, confirming their butanol-rich composition [Fig. 3(b)]. The area outside the droplets mainly exhibits $\mathrm{CH}_{3}$ symmetric stretching vibration from DMSO, also accompanied by features from a small amount of miscible butanol (as indicated by a shoulder peak at $2873 \mathrm{~cm}^{-1}$ and relatively broad peak at $2925 \mathrm{~cm}^{-1}$ ). Based on the SRS intensity at $2873 \mathrm{~cm}^{-1}$, the butanol concentration outside the droplets is estimated to be $4-5$ times less than the droplet interior. 
To measure the H-bonding environment in these droplets, a hyperspectral stack of 35 images was collected from $2211 \mathrm{~cm}^{-1}$ to $2260 \mathrm{~cm}^{-1}$ at $10 \mathrm{~s}$ per frame under pump power of $15 \mathrm{~mW}$ and Stokes power around 17 $\mathrm{mW}$. A major benefit of using a nitrile probe is the near-zero background from other vibrations in this region, as demonstrated by the lack of any features when probing the nitrile region without Rhod 800 present [Fig. 3(c)]. Figure 3(e) shows a representative SRS frame at $2230 \mathrm{~cm}^{-1}$, indicating a higher concentration of Rhod800 within the droplets due to higher butanol concentration. Since these are hsSRS data, each pixel contains an entire spectrum that can be used to examine small peak shifts. Figure 3(d) gives two representative spectra for the pixels inside and outside the droplets. A $5 \times 5$ pixel spatial filter was applied to improve the signal-to-noise ratio. To obtain the spatial distribution of the nitrile shift, the peak maximum was found at each pixel by using a Lorentzian fit as detailed above. As can be seen from the reconstructed frequency colormap [Fig. 3(f)], the nitrile probe reports a lower nitrile frequency inside the butanol microdroplets compared to the water surroundings, indicating a consistent trend with bulk measurement in terms of local solvation, but with sub-micron spatial resolution, even within crowded areas [Fig. 3(i-j)]. 
(a)
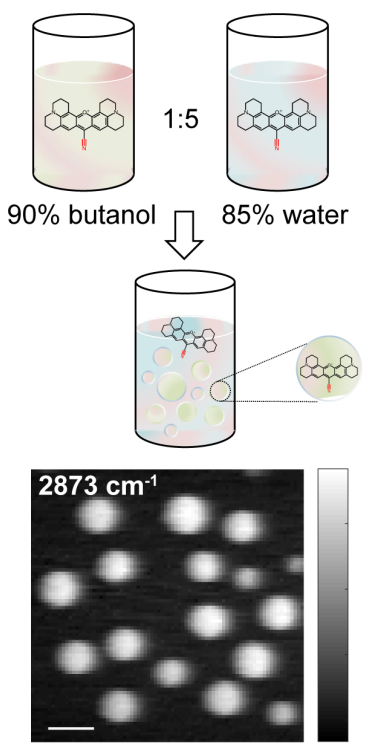

Butanol droplets in water

(e)

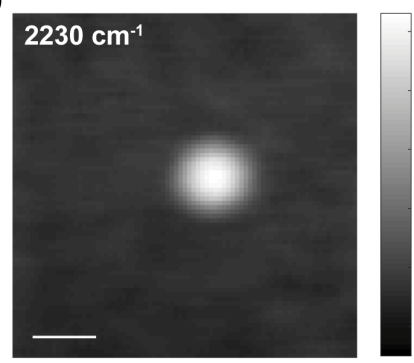

(f)

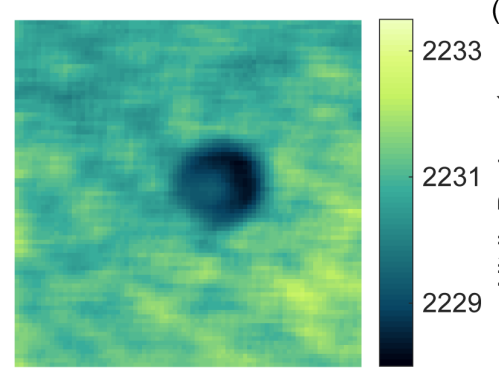

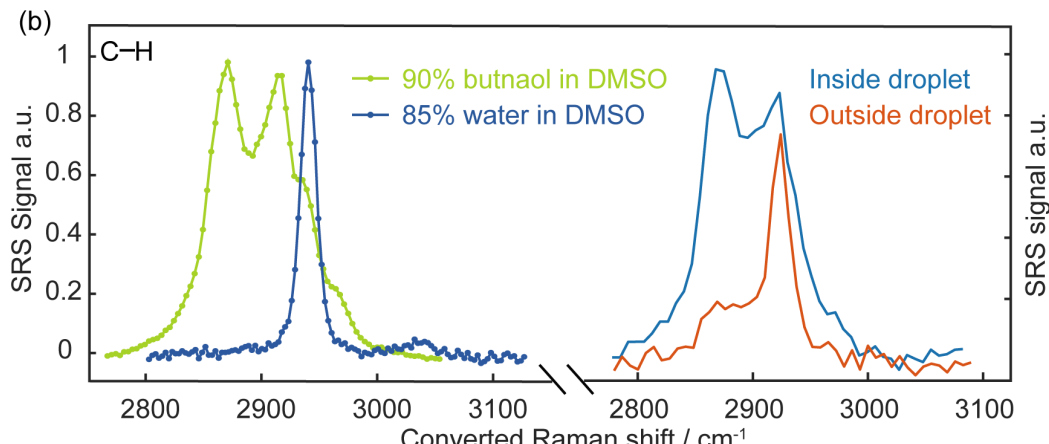

(c)
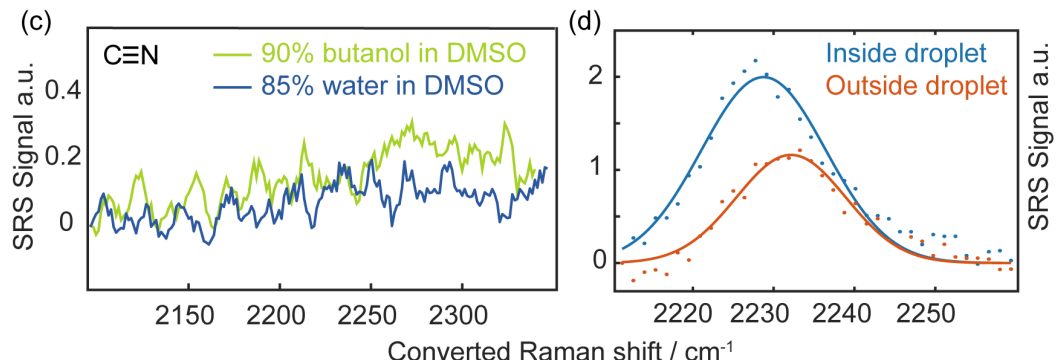

$\begin{array}{llll}2220 & 2230 & 2240 & 2250\end{array}$ (g)

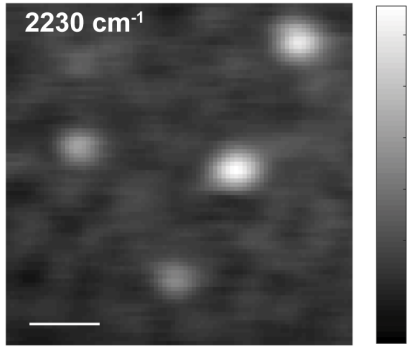

(h)

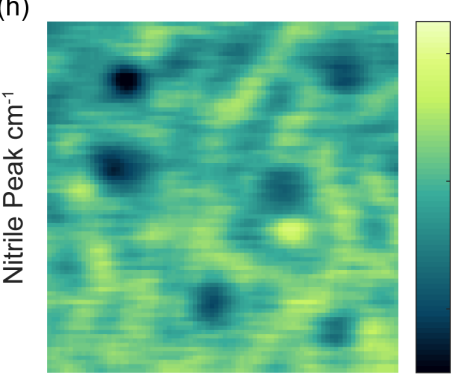

(i)

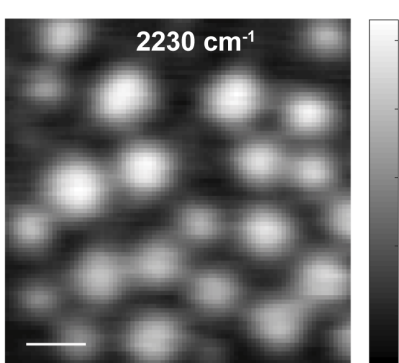

(j) 2233

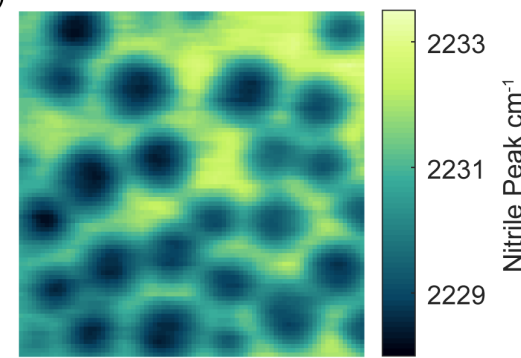

Figure 3. Nitrile-shift imaging of microdroplets with hsSRS microscopy. (a) Process-flow for generating butanolwater droplets containing Rhod800. Bottom: SRS image of emulsions at $2873 \mathrm{~cm}^{-1}$ (CH-stretching). (b-d) Comparison of hsSRS spectra in the $\mathbf{C}-\mathbf{H}$ region (b) and the $\mathbf{C} \equiv \mathbf{N}$ region (c-d) for bulk solutions and droplet emulsions. Left: $90 \%$ butanol in DMSO (green) and 85\% water in DMSO (blue). Right: pixels inside droplets (light blue) outside droplets (red). (e-i) Representative SRS images of droplets at nitrile frequency $2230 \mathrm{~cm}^{-1}$ (f-j) Corresponding nitrile frequency mapping from hsSRS scans. 35 sequential frequency steps from $2218 \mathrm{~cm}^{-1}$ to $2243 \mathrm{~cm}^{-1}$ with $1.4 \mathrm{~cm}^{-1}$ intervals were scanned with $1 \mathrm{~ms}$ pixel dwell time. $P_{\text {pump }}=15.4 \mathrm{~mW}, P_{\text {Stokes }}=17.4 \mathrm{~mW}(\mathrm{f}-\mathrm{h}) . \mathrm{P}_{\text {pump }}=12 \mathrm{~mW}, \mathrm{P}_{\text {Stokes }}=12.2 \mathrm{~mW}$ (j). Scale bar $=5 \mu \mathrm{m}$. 


\section{Probing cellular solvation heterogeneities with hsSRS}

Having demonstrated that this method can be used to sense solvation at the microscopic level, we further sought to map and quantify solvation in the live cell environment. To do so, Rhod800 was introduced to live HeLa cells to probe the H-bonding character of different areas of the subcellular environment. As a control, HeLa cells treated only with DMSO/PBS show no nitrile signal at $2230 \mathrm{~cm}^{-1}$ [Fig. 4(a)], corroborating the low endogenous background in this region of the spectrum. Different staining concentrations of dye $(1 \mu \mathrm{M}$ to $100 \mu \mathrm{M}$, Fig. S6) were tested to obtain a uniform distribution of the dye. Cells treated with $5 \mu \mathrm{M}$ Rhod800 show enrichment of dyes inside endosomes (evidenced by the appearance of puncta in the cytosol) with very little incorporation of dyes in the nucleus, while $10-50 \mu \mathrm{M}$ Rhod800 treated cells exhibit a ubiquitous distribution of dyes, with a higher concentration in the nucleoli (Fig. S7). Even higher concentration of Rhod800 $(100 \mu \mathrm{M})$ causes solid aggregates inside the cells. Therefore, all subsequent cell imaging was carried out at a staining concentration less than $40 \mu \mathrm{M}$ to maintain appropriate $\mathrm{S} / \mathrm{N}$ and avoid aggregation. Approximately $10 \mathrm{~mW}$ of the pump and the Stokes beam were used for live cell imaging. Figure 4(b) shows a representative SRS image of an isolated cell in the nitrile region. As the SRS signal has a linear concentration dependence, we can estimate the local dye concentration inside the cells based on calibration of dye solutions at known concentrations. Using this approach, Rhod800 was found to be twice as concentrated in nucleoli as compared to the cytoplasm and nucleus, which had an average dye concentration of $1.5-3.5 \mathrm{mM}$ [Fig. S8(f)].

Examining the nitrile-shift map reveals a striking heterogeneity in peak position as a function of subcellular location. The nitrile frequency map of Rhod 800 treated HeLa cells was determined following the procedure outlined above, with a few representative mapping results shown in Fig. 4(b-c) and Fig. S9. The results are discussed separately below. 

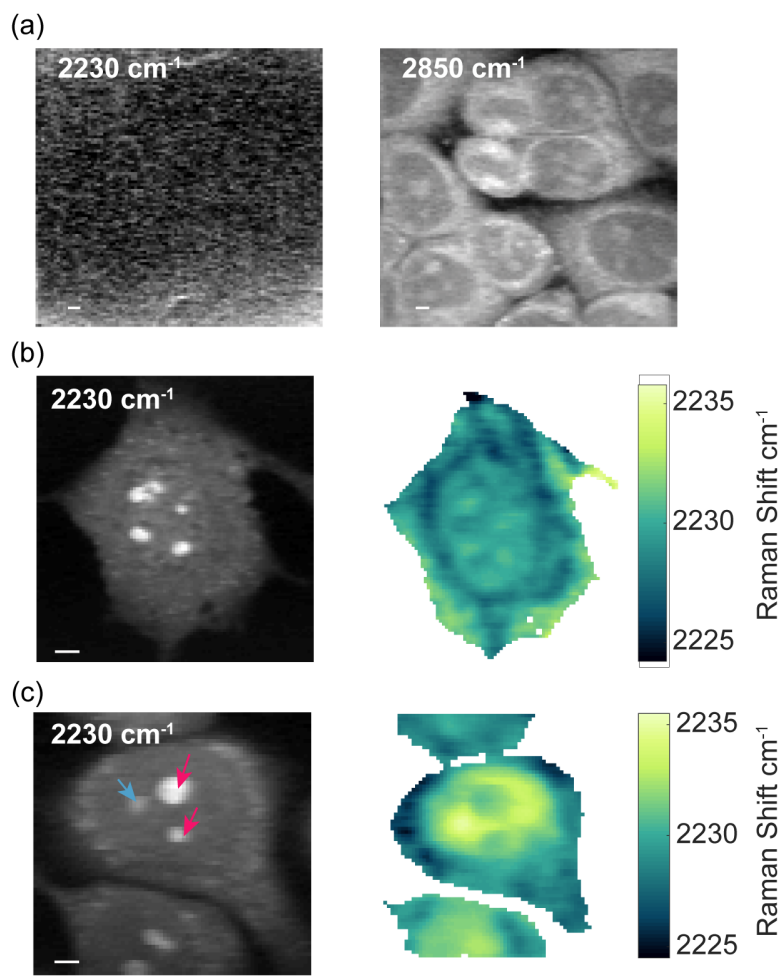

Figure 4. Nitrile-shift imaging of HeLa cells with hsSRS microscopy. (a) SRS images of HeLa cell without Rhod800 treatment. Left: at $2230 \mathrm{~cm}^{-1}$ (nitrile); Right: at $2850 \mathrm{~cm}^{-1}$ (lipid). (b-c) Left: Representative SRS image at $2230 \mathrm{~cm}^{-1}$ of HeLa cell stained with $10 \mu \mathrm{M}$ Rhod800 for 30 mins. 18 sequential frequency steps from $2218 \mathrm{~cm}^{-1}$ to $2243 \mathrm{~cm}^{-1}$ with $1.4 \mathrm{~cm}^{-1}$ intervals were scanned under $8.7 \mathrm{~mW}$ (pump power) and $10.4 \mathrm{~mW}$ (Stokes power) with 200 $\mu$ s pixel dwell time (b, 10 frames averaging) and $400 \mu \mathrm{s} \mathrm{(c,} 5$ frames averaging). Right: Corresponding nitrile-shift mapping of cells, which show $\omega_{\text {cytoplasm }}<\omega_{\text {nucleus }}<\omega_{\text {nucleoli }}\left(\mathrm{b}\right.$ and areas pointed by blue arrow in $\mathrm{c}$ ); or $\omega_{\text {cytoplasm }}$ $\leq \omega_{\text {nucleoli }}<\omega_{\text {nucleus }}$ (areas indicated by pink arrows in c). Scale bar $=5 \mu \mathrm{m}$.

Cytoplasm and Nucleus: A marked and consistent blue-shift is observed in the nucleus relative to the cytoplasm $\left(\omega_{\text {cytoplasm }}<\omega_{\text {nucleus }}\right)$ among $98 \%$ cells $(\mathrm{N}>100)$, showing increased solvation and H-bonding in the nucleus. A wide range of controls were investigated in order to rationally interpret this frequency trend in the context of solvation environments. To isolate the effect of H-bonding from other factors that might exist within the complex cellular interior, the nitrile peak shift was measured as a function of $\mathrm{pH}$, probe concentration and ionic strength. The nitrile peak of Rhod800 is nearly $\mathrm{pH}$-insensitive (only $0.5 \mathrm{~cm}^{-1}$ shift over $\mathrm{pH}$ 3-10) and exhibits negligible concentration-dependence under the concentrations found inside cells [Fig. S10(a-b)]. A red-shift $\left(0.4 \mathrm{~cm}^{-1}\right)$ is observed under the intracellular ion concentration which is estimated to be less than $200 \mathrm{mM}$ [Fig. S10(c)]. ${ }^{54}$ However, there is no evidence suggesting a large difference in salt concentration between cytoplasm and nucleus. Given the relatively small scale of the shift 
over a large range of salt concentrations, we believe that this cannot be responsible for the observed difference in nitrile shift between cytoplasm and nucleus.

It is important to note that dyes mainly exist as monomers in DMSO where conjugated ring structure of the Rhod800 molecule is well-solvated. Dimers and even aggregates can be formed when increasing the fraction of water in the binary mixture or increasing dye concentration in water (saturated concentration around $83 \mu \mathrm{M})^{55}$, as confirmed by the ratio change between two absorption peaks (Fig. S11). The aggregated state can result in a red-shift in the nitrile frequency as compared to the well-solvated nitrile tag. A red-shift around $1.5 \mathrm{~cm}^{-1}$ is observed for aggregates inside butanol-water emulsions, with SRS signal 5 times higher than the surrounding solution (Fig. S12). In cells, although we estimated the cellular concentration of the dye to be in the order of mM, we didn't observe obvious aggregation inside cells, as evidenced by the uniform signal level between cytosol and nucleus [Fig. 4(b)]. This suggests that the cell environment is not equivalent to the dilute aqueous solution, and Rhod800 is more soluble in the cytosol and nucleus than it is in pure water. From this perspective, the cellular interior is more similar to DMSO rather than bulk water with regards to the increased molecular solubility and reduced nitrile solvation. Within the extreme crowding cellular environment, it is possible that some interactions with biomolecules contribute to this phenomenon. In this study, a similar concentration of Rhod800 between the cytosol and nucleus is observed [Fig. S8(e)], suggesting that the concentration or potential "aggregation effect" is not a concern since it cannot explain the large shift seen between the two environments.

In addition to the above potential environmental effects, it is critical to characterize the photo-stability of the dyes used for pre-resonance SRS because this hsSRS method closely correlates the Raman shift with time delay. Nitrile signal in solution-phase and micro-droplets measurements remain relatively stable even measured under higher laser power or longer acquisition window due to effective thermal dissipation [Fig. S13(a-c)]. On the other hand, obvious photobleaching was observed in consecutive cell scans [Fig. S13(de)]. Nitrile photostability inside cells was tested by collecting time series data at $2230 \mathrm{~cm}^{-1}$ for $300 \mathrm{~s}$ (Fig. 
S14). In these data, photo-bleaching was generally observed for SRS signals in both the cytoplasm and nucleus, with the decay in the cytoplasm occurring at a slightly faster rate. This bleaching behavior, or any other time-dependent intensity fluctuation, could be problematic during a relatively longer acquisition window (4 - 5 mins) because it may result in artifacts and complicate data interpretation. To understand this effect, we can consider the experimentally observed Raman shift as the combination of an intrinsic symmetric Raman peak affected by a time-correlated signal fluctuation. Under forward scanning, spectra are acquired by scanning the delay stage from low wavenumber to high wavenumber, the overall photobleaching trend can cause an asymmetric spectrum with a downward shape (modeled as blue curve in Fig S15). As a result, a red-shifted peak position would be obtained upon fitting. However, this artifact can be reversed as a blue-shifted peak by simply switching the scanning direction, where the spectra are acquired from high to low wavenumber (backward scan). This simple model is consistent with the trend that higher nitrile frequency usually appeared under backward scans [Fig. 5(c-d)] compared to forward scans [Fig. 5(a-b)].

By comparing the spectra obtained from both scanning directions, the underlying vibrational shift could be disentangled from the time-dependent bleaching effect. With bi-directional scanning, we note the nitrile frequency of dyes inside the nucleus is always blue-shifted compared to the cytosolic dyes $\left(\omega_{\text {nucleus }}>\right.$ $\left.\omega_{\text {cytoplasm }}\right)$ regardless of the magnitude of the frequency differences [Fig. 5(a-d)]. This reveals that the nitrile blue-shift is due to a real difference in the local solvation and not an artifact of photobleaching. To evaluate this effect at the population level, we extracted the average nitrile frequency from the entire ROI of the cytoplasm and the nucleus inside one cell and calculated the difference $\left(\Delta \omega \mathrm{cm}^{-1}\right)$ between them. By analyzing over one hundred different cells, we generated the distribution of $\Delta \omega$ for both forward and backward scans [Fig. 5(f)]. The forward scan shows a $1.89 \pm 0.77 \mathrm{~cm}^{-1}$ blue-shift between cytoplasm and nucleus, while the backward scan yields $1.29 \pm 0.37 \mathrm{~cm}^{-1}$. This is consistent with our model that faster decay of the SRS signal in the cytoplasm can cause the nitrile frequency to red-shift more than the nucleus in the forward scan and blue-shift more in the backward scan. The average value $\left(1.59 \pm 0.43 \mathrm{~cm}^{-1}\right)$ is used 
to represent the actual nitrile shift caused by local solvation environments. A similar approach applied to the raw peak positions yields an average nitrile frequency of $2229.48 \pm 0.97 \mathrm{~cm}^{-1}\left(\omega_{\text {cytoplasm}}\right)$ and $2231.34 \pm$ $0.96 \mathrm{~cm}^{-1}\left(\omega_{\text {nucleus }}\right)$. These peak positions represent the overall response to the variations of the local solvation environment, including both the local electrostatics and H-bonding. The latter is the predominant factor dictating the heterogeneity of nitrile frequency inside the cells. This is also validated by the fieldfrequency diagram from solution-phase solvatochromic measurements. The electrostatic effect on nitrile frequency lies in a relatively small range of $2228.5 \mathrm{~cm}^{-1}$ (DMSO) to $2230.5 \mathrm{~cm}^{-1}$ (chloroform) while the Hbonding ( $85 \%$ water) results in a range of $4.7 \mathrm{~cm}^{-1}$ blue shift [Fig. S4(f)].

To gain quantitative insight into the local solvation environment, the averaged nitrile frequency can be mapped onto the water-content calibration curve obtained in solution phase [Fig. 5(h)]. While DMSO-water mixtures might be too simple to simulate the intracellular environment, it still represents a good control on testing the solvation effect for polar environments and gives us an approximate idea of how the solvation environment vary in subcellular compartments. The results shift slightly if DMF-water mixtures are chosen as the reference. Upon DMSO-water calibration, this nitrile frequency yields an estimated 29.5\% (S.E.: $1.8 \%$ ) free water content in the cytoplasm and $57.3 \%$ (S.E.: $1.0 \%$ ) free water content in the nucleus. This is in very strong agreement with the values found by Min and co-workers using Raman-modulated fluorescence $^{46}$ [36\% (cytosol) vs. $65 \%$ (nucleus) free water contents in single HeLa cell]. When compared with literature values reported for oocytes using NMR microscopy, ${ }^{10}$ we found some difference in the magnitude of solvation within different cellular compartments, but a similar trend was observed with the nucleus exhibiting more free water (89.4\%) compared to the animal pole (74.4\%) and vegetal pole (44.5\%). Taken together, this work, along with these other investigations, indicates drastically different solvation environments between the cytoplasm and the nucleus. The relatively low amount of solvation observed in the cytosol can be understood from the perspective of macromolecular crowding. The surface of proteins is known to be covered in 2-10 layers of bound water, which behave differently than bulk water and are likely not to participate in H-bonding to the same extent. ${ }^{56}$ Estimated protein concentrations within cells 
$(200-300 \mathrm{mg} / \mathrm{mL})^{57}$ suggest that there may be limited room for bulk water, resulting in the data shown in Fig. 5(h).

(a)
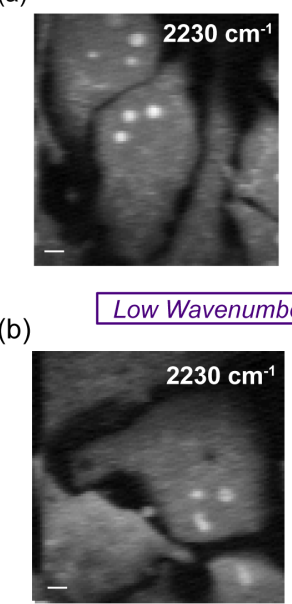

(e)

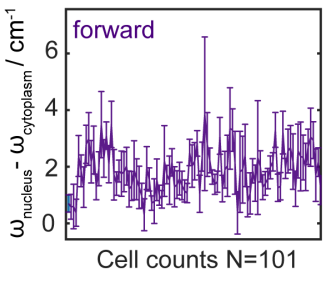

(f)

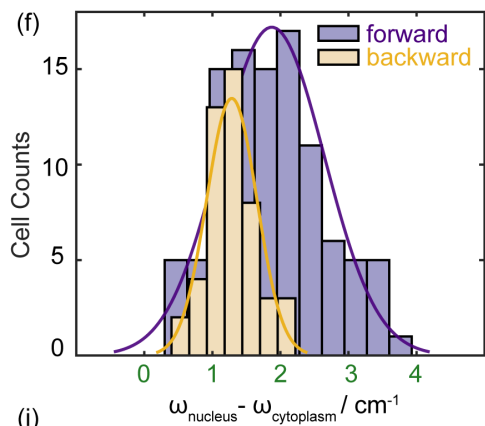

(i)
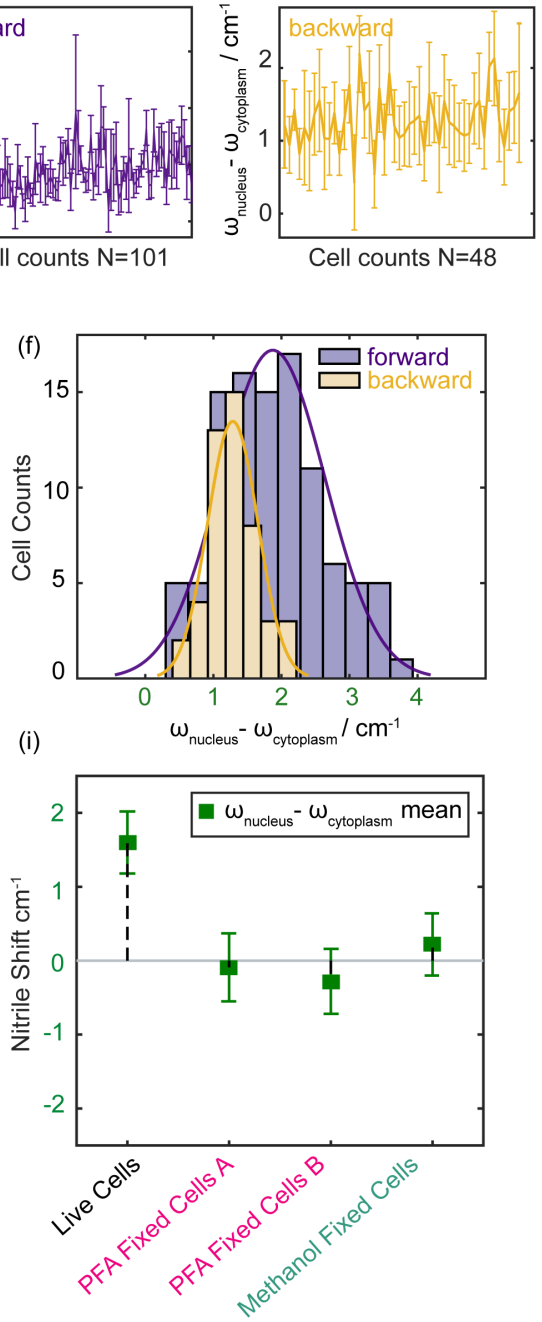

(c)
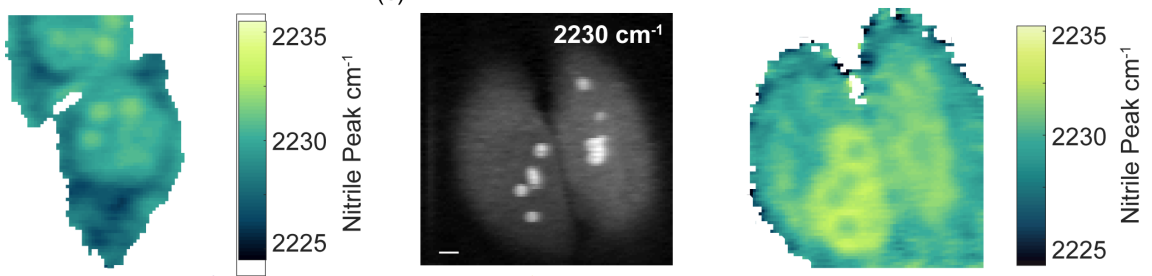

(d)
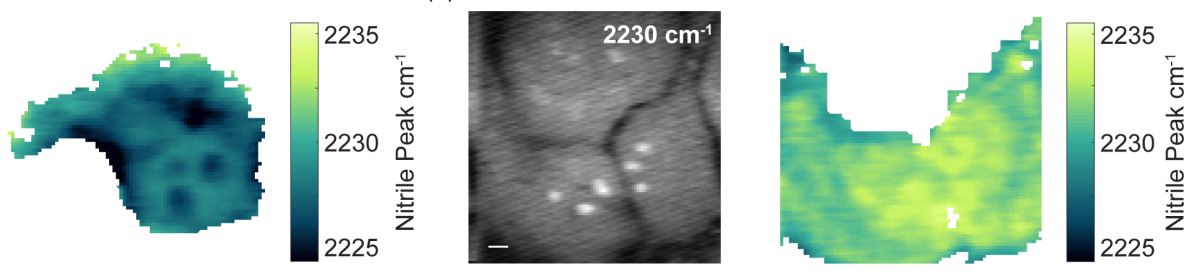

(g)
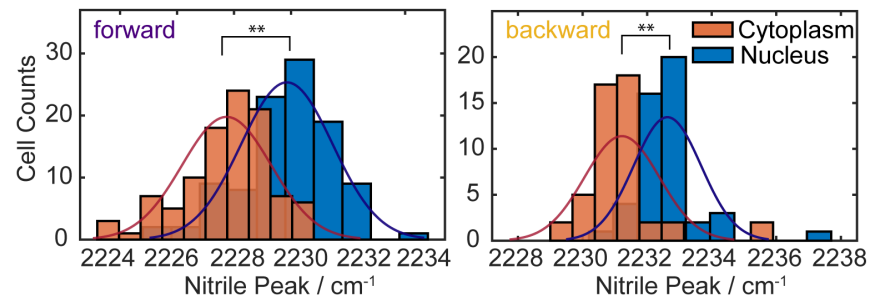

(h)

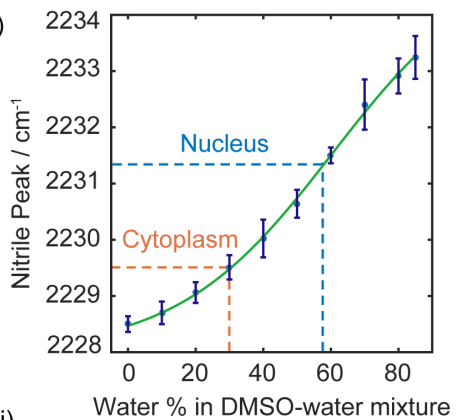

(j)

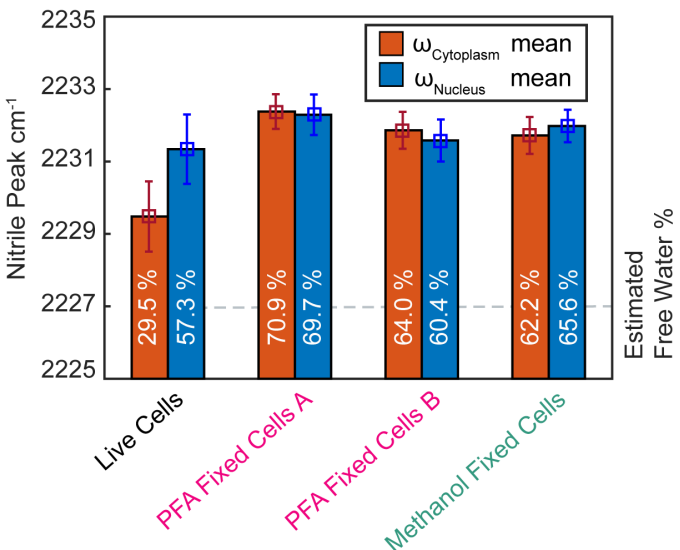


Figure 5. Quantitative analysis of solvation heterogeneity inside HeLa cells by two-ways hsSRS scan. Representative SRS images and corresponding nitrile shift mapping of cells under forward scan (a-b) and backward scan (c-d). Scale bar $=5 \mu \mathrm{m}$. (e) Scatter plots of nitrile frequency difference $(\Delta \omega)$ between the cytoplasm and nucleus under forward scans (purple, 101 cells) backward scans (yellow, 48 cells). (f) Histograms of $\Delta \omega$. Normal distribution fitting shows $\Delta \omega=1.89 \mathrm{~cm}^{-1} \pm 0.77 \mathrm{~cm}^{-1}$ (forward); $\Delta \omega=1.29 \mathrm{~cm}^{-1} \pm 0.37 \mathrm{~cm}^{-1}$ (backward). Mean: $\Delta \omega=1.59 \mathrm{~cm}^{-1} \pm$ $0.43 \mathrm{~cm}^{-1}$. (g) Histograms of nitrile frequency in cytoplasm and nucleus regions. Forward scan: $\omega_{\text {cytoplasm }}=2227.74$ $\mathrm{cm}^{-1} \pm 1.56 \mathrm{~cm}^{-1} ; \omega_{\text {nucleus }}=2229.85 \mathrm{~cm}^{-1} \pm 1.61 \mathrm{~cm}^{-1}$; Backward scan: $\omega_{\text {cytoplasm }}=2231.22 \mathrm{~cm}^{-1} \pm 1.16 \mathrm{~cm}^{-1} ; \omega_{\text {nucleus }}$ $=2232.83 \mathrm{~cm}^{-1} \pm 1.05 \mathrm{~cm}^{-1}$; Mean: $\omega_{\text {cytoplasm }}=2229.48 \pm 0.97 \mathrm{~cm}^{-1} ; \omega_{\text {nucleus }}=2231.34 \mathrm{~cm}^{-1} \pm 0.96 \mathrm{~cm}^{-1}$. Statistically significant differences exist between the cytoplasm and nucleus. $* *$ indicates $\mathrm{p}<0.001$ from a two-tailed $t$-test, (h) Estimation of free water content in the cytoplasm and nucleus based on the nitrile shift calibration curve (Sigmoidal fitting) obtained with DMSO-water mixtures. Cytoplasm [29.5\% (1.5\%-45.3\%), S.E. : 1.8\%] vs. Nucleus [57.3\% (44.3\%-69.8\%), S.E. : 1.0\%). (i) $\Delta \omega$ comparison between live cells and fixed cells by averaging two-ways scans. (j) Averaged nitrile frequency of cytoplasm and nucleus in live cells and fixed cells. Free water percentage is calculated by projecting averaged $\omega_{\text {cytoplasm }}$ and $\omega_{\text {nucleus }}$ onto the calibration curve $(h)$.

To validate this hypothesis, similar measurements were performed on fixed HeLa cells. Different fixatives [paraformaldehyde (PFA), and methanol] and slightly different fixation order are used as comparison. Relatively higher power condition (Pump=20 mW, Stokes=33 mW) were used to maintain good S/N. The estimated dye concentration is about 1-1.5 mM inside cells with the Rhod800 slightly more concentrated in the cytoplasm than the nucleus [Fig. S16]. Bi-directional scans were performed on each group to compensate for the bleaching effect [Fig. S17, S18]. In contrast to live cells, fixed cells exhibit a homogenous frequency pattern over different cell compartments. The average nitrile shift $(\Delta \omega)$ between the cytosol and nucleus in fixed cells are $\left(-0.09 \pm 0.46 \mathrm{~cm}^{-1}\right),\left(0.22 \pm 0.42 \mathrm{~cm}^{-1}\right)$ and $\left(-0.28 \pm 0.44 \mathrm{~cm}^{-1}\right)$ for PFA fixed group A, group B and methanol fixed group, respectively [Fig. 5(i)]. Compared to a $1.59 \mathrm{~cm}^{-1}$ shift in live cells, these small variations between cytosol and nucleus suggest the solvation heterogeneity is significantly minimized through fixation. It should be noted that the cytoplasm and nucleus are affected differently with regard to the change in cell state [Fig. 5(j)]. The average nitrile frequency in the cytosol increases drastically about $2.9 \mathrm{~cm}^{-1}$ (PFA group A), $2.4 \mathrm{~cm}^{-1}$ (PFA group B) and $2.4 \mathrm{~cm}^{-1}$ (methanol group) from $2229.48 \mathrm{~cm}^{-1}$ while nitriles in nucleus show a relatively small increase $\left(0.24 \mathrm{~cm}^{-1}-0.95 \mathrm{~cm}^{-1}\right)$ from $2231.34 \mathrm{~cm}^{-1}$ upon fixation. These results support our attribution of lower nitrile frequency in cytosol to lower ratio of free water due to the macromolecular crowding. In fixed cells, paraformaldehyde crosslinks molecules inside cells by reacting with the primary amine in proteins while the alcohol-based process 
denatures proteins. Both fixatives disrupt the solvation shell around proteins and increase the amount of water available for $\mathrm{H}$-bonding. As a result, there is more free water inside the cytosol, leading to an increase from $29.5 \%(1.5 \%-45.3 \%)$ in live cells to $70.9 \%(64.5 \%-77.7 \%), 64.0(57.4 \%-70.8 \%)$, and $62.2 \%(55.6 \%$ $68.9 \%$ ) in fixed groups. It's interesting to note that methanol-based fixation also removes lipids in cells but causes similar results compared to PFA group B where the lipids are maintained intact. This suggests that lipids (electrostatic contribution) play a minor role on the observed nitrile shift.

To further prove this hypothesis in vitro, the nitrile shift of Rhod800 as a function of BSA concentration are examined. As shown in the Fig. S19(a), a nitrile red-shift $\left(1.5 \mathrm{~cm}^{-1}\right)$ is observed when increasing the concentration of BSA to $300 \mathrm{mg} / \mathrm{mL}$ (close to the estimated concentration of protein in the cytosol). As increasing concentrations of BSA, there is less free water contributing to nitrile solvation as water forms H-bonds with BSA. To further confirm that this nitrile shift is uncoupled from other interaction between Rhod800 molecules and BSA, we measured the nitrile shift of acetonitrile in the presence of BSA [Fig. S19 (b)]. About $1 \mathrm{~cm}^{-1}$ red-shift was obtained at similar BSA concentrations, indicating this phenomenon is exclusively attributable to nitrile solvation. These in vitro experiments reproduce the observed results in the cytoplasm simply by using a comparable concentration of protein. We believe that this supports our hypothesis that the observed nitrile shift in the cytoplasm is due to macromolecular crowding.

Nucleoli: In contrast to the consistent blue-shift between the cytoplasm and the nucleus, the nitrile frequency associated with nucleoli appeared to vary from cell to cell. In some cases, as depicted in Fig. 4(b) [and Fig. S9(a-d)], nucleoli exhibits the largest blue-shift (highest nitrile frequency) compared to the rest of the cell, while in other cases, it exhibits similar or even red-shifted frequency compared to the nucleus [Fig. S9(e-h)]. There is no morphological correlation among cells with different observed $\omega_{\text {nucleoli. }}$ It is even observed that nucleoli in a single cell show different peak positions [Fig. 4(c), pink and blue arrows]. The fact that the underlying properties of individual nucleoli inside one cell are not likely to vary suggests the different behavior of $\omega_{\text {nucleoli }}$ may be manipulated by other factors besides subcellular variability. 
Aggregation of dyes likely exists in the nucleoli and that may be associated with the unusual signal fluctuation in the time-series data (Fig. S14). The signal was found to be enhanced at the beginning [Fig. S14(a), possibly due to dye accumulation], followed by the fast bleaching [Fig. S14(c)]. According to our simple model, if the imaging stack only captures the signal increase inside nucleoli, the observed frequency would be overestimated in a low-to-high wavenumber scan [Fig. 5(a)]. If only bleaching occurs, the frequency would be underestimated in a forward scan [Fig. 5(b)]. This likely explains the often-conflicting behavior of nucleoli detailed above, where observed nitrile shift results from a change in the SRS intensity rather than an actual shift in the vibrational character. Similarly, by switching to high-to-low wavenumber scan, the reversed trend of nitrile frequency inside the nucleoli is observed [Fig. 5(c-d)]. These data suggest that the peak shift data from nucleoli in these experiments are not a good readout of local dye solvation.

\section{Comparison with previous work}

At the time of the submission of this work, Min and co-workers reported the use of stimulated Raman enhanced fluorescence (SREF) as a method to apply the "nitrile shift" idea to probe the intracellular water heterogeneity. ${ }^{47}$ SREF uses a fluorescence readout rather than directly measuring the SRS signal, but the principle is the same. They measured a distinct difference in the solvation between the cytosol and nucleus [36\% (cytosol) vs. $65 \%$ (nucleus), compared to $29.5 \pm 1.8 \%$ (cytosol) free water content in the cytoplasm and $57.3 \pm 1.0 \%$ (nucleus) in the current work]. Critically, both studies reveal reduced solvation in live cells and differing solvation environments between the cytosol and nucleus. Table S4 summarized the experimental parameters between these two methods. The SREF method shows better sensitivity (nM) due to fluorescence detection, however, the current work demonstrates that such sensitivity is not necessarily required and solvation can be probed without relying on fluorescence detection. We believe the current work is valuable in demonstrating the power of the hyperspectral SRS, which is a less complicated optical setup, in imaging microscopic differences in solvation with comparable speed and resolution of the SREF work. Another advantage over SREF in vibrational shift imaging is our hsSRS approach doesn't require the 
probe to be a fluorophore, which expands the application scope in probe designs. For example, this could potentially apply to molecules which are good chromophores but with low quantum yields.

\section{Conclusions}

The distinct solvation variance between the cytoplasm and nucleus observed here is in substantial agreement with prior studies (both $\mathrm{SREF}^{47}$ and NMR microscopy ${ }^{10}$ ). Taken together, these results provide new insights into the solvation heterogeneity in different cellular compartments. These foundational experiments suggest further study is needed to investigate how solvation varies not just between the nucleus and the cytoplasm but within other cellular sub-structures. Future studies can begin to examine how these solvation differences affect local biochemistry. Importantly, this demonstration also provides a brand-new perspective to study biological liquid-liquid phase separation, ${ }^{58}$ where more quantitative assays and descriptions are needed to probe the physical and chemical principles of membrane-less condensates. These critical studies will require further advancement in instrumentation and probe design to increase sensitivity. These ends could potentially be achieved by rationally designing a sensitive nitrile probe away from resonance conditions and speeding up data acquisition, including employing a faster lock-in amplifier and a resonant mirror $^{59}$ to improve the acquisition time for hyperspectral imaging.

\section{Supplementary Material:}

Additional details of the experiments, image processing methods and additional figures are presented in the supplemental material.

\section{References:}

1. P. Ball, Chem. Rev. 108 (1), 74-108 (2008).

2. M. Chaplin, Nature Reviews Molecular Cell Biology 7 (11), 861-866 (2006).

3. K. Luby-Phelps, Molecular Biology of the Cell 24 (17), 2593-2596 (2013).

4. J. S. Clegg, J. Cell Biol. 99 (1), S167-S171 (1984).

5. J. Israelachvili and H. Wennerström, Nature 379 (6562), 219-225 (1996).

6. E. Odeblad, B. N. Bhar and G. Lindström, Arch. Biochem. Biophys. 63 (1), 221-225 (1956).

7. C. F. Hazlewood, B. L. Nichols and N. F. Chamberlain, Nature 222 (5195), 747-750 (1969). 
8. C. B. Bratton, A. L. Hopkins and J. W. Weinberg, Science 147 (3659), 738-739 (1965).

9. S. R. Kasturi, P. K. Seitz, D. C. Chang and C. F. Hazlewood, Biophys. J. 58 (2), 483-491 (1990).

10. S. Pauser, A. Zschunke, A. Khuen and K. Keller, Magn. Reson. Imaging 13 (2), 269-276 (1995).

11. E. A. López-Beltrán, M. J. Maté and S. Cerdán, J. Biol. Chem. 271 (18), 10648-10653 (1996).

12. T. Tsukahara, A. Hibara, Y. Ikeda and T. Kitamori, Angew. Chem. Int. Ed. 46 (7), 1180-1183 (2007).

13. C. Gabriel, R. J. Sheppard and E. H. Grant, PMB 28 (1), 43-49 (1983).

14. P. Pissis, A. Anagnostopoulou-Konsta and L. Apekis, Europhysics Letters (EPL) 3 (1), 119-125 (1987).

15. R. Sampathkumar, D. Gopalakrishnan and A. C. Kumbharkhane, Int. J. Biol. Macromol. 118, 1811-1816 (2018).

16. E. C. Trantham, H. E. Rorschach, J. S. Clegg, C. F. Hazlewood, R. M. Nicklow and N. Wakabayashi, Biophys. J. 45, 927-938 (1984).

17. A. M. Stadler, J. P. Embs, I. Digel, G. M. Artmann, T. Unruh, G. Büldt and G. Zaccai, J. Am. Chem. Soc. 130 (50), 16852-16853 (2008).

18. F. Foglia, R. Hazael, G. G. Simeoni, M.-S. Appavou, M. Moulin, M. Haertlein, V. Trevor Forsyth, T. Seydel, I. Daniel, F. Meersman and P. F. McMillan, Scientific Reports 6 (1), 18862 (2016). 19. M. P. M. Marques, A. L. M. Batista de Carvalho, V. G. Sakai, L. Hatter and L. A. E. Batista de Carvalho, PCCP 19 (4), 2702-2713 (2017).

20. $\quad$ S. Woutersen and H. J. Bakker, Phys. Rev. Lett. 83 (10), 2077-2080 (1999).

21. S. Woutersen, U. Emmerichs and H. J. Bakker, Science 278 (5338), 658-660 (1997).

22. M. C. Thielges, D. A. Case and F. E. Romesberg, J Am Chem Soc 130 (20), 6597-6603 (2008).

23. L. B. Sagle, J. Zimmermann, P. E. Dawson and F. E. Romesberg, J. Am. Chem. Soc. 128 (44), 14232-14233 (2006).

24. C. S. Miller and S. A. Corcelli, J. Phys. Chem. B 114 (25), 8565-8573 (2010).

25. S. X. Ye, T. Huber, R. Vogel and T. P. Sakmar, Nat Chem Biol 5 (6), 397-399 (2009).

26. S. D. Fried, S. Bagchi and S. G. Boxer, Science 346 (6216), 1510-1514 (2014).

27. S. D. Fried, S. Bagchi and S. G. Boxer, J Am Chem Soc 135 (30), 11181-11192 (2013).

28. J. T. King and K. J. Kubarych, J. Am. Chem. Soc. 134 (45), 18705-18712 (2012).

29. A. T. Fafarman and S. G. Boxer, J Phys Chem B 114 (42), 13536-13544 (2010).

30. B. A. Lindquist, K. E. Furse and S. A. Corcelli, Phys Chem Chem Phys 11 (37), 8119-8132 (2009).

31. J. Zimmermann, M. C. Thielges, Y. J. Seo, P. E. Dawson and F. E. Romesberg, Angew Chem Int Edit 50 (36), 8333-8337 (2011).

32. S. K. Jha, M. B. A. Ji, K. J. Gaffney and S. G. Boxer, Proc. Natl. Acad. Sci. U.S.A. 108 (40), 16612-16617 (2011).

33. R. Adhikary, J. Zimmermann and F. E. Romesberg, Chem Rev 117 (3), 1927-1969 (2017).

34. I. T. Suydam and S. G. Boxer, Biochemistry 42 (41), 12050-12055 (2003).

35. A. J. Stafford, D. M. Walker and L. J. Webb, Biochemistry 51 (13), 2757-2767 (2012).

36. M. C. Thielges, J. Y. Axup, D. Wong, H. S. Lee, J. K. Chung, P. G. Schultz and M. D. Fayer, J. Phys. Chem. B 115 (38), 11294-11304 (2011).

37. A. T. Fafarman, P. A. Sigala, D. Herschlag and S. G. Boxer, J Am Chem Soc 132 (37), 1281112813 (2010).

38. M. N. R. Johnson, C. H. Londergan and L. K. Charkoudian, J Am Chem Soc 136 (38), 1346813468 (2014).

39. Z. Getahun, C. Y. Huang, T. Wang, B. De Leon, W. F. DeGrado and F. Gai, J Am Chem Soc 125 (2), 405-411 (2003).

40. P. A. Sigala, A. T. Fafarman, P. E. Bogard, S. G. Boxer and D. Herschlag, J Am Chem Soc 129 (40), 12104-+ (2007).

41. P. Deb, T. Haldar, S. M. Kashid, S. Banerjee, S. Chakrabarty and S. Bagchi, J Phys Chem B 120 (17), 4034-4046 (2016). 
42. M. G. Maienschein-Cline and C. H. Londergan, J. Phys. Chem. A 111 (40), 10020-10025 (2007).

43. A. F. Palonpon, M. Sodeoka and K. Fujita, Curr Opin Chem Biol 17 (4), 708-715 (2013).

44. $\quad$ R. Smith, K. L. Wright and L. Ashton, Analyst 141 (12), 3590-3600 (2016).

45. H. Yamakoshi, A. F. Palonpon, K. Dodo, J. Ando, S. Kawata, K. Fujita and M. Sodeoka, Chem. Commun. 50 (11), 1341-1343 (2014).

46. F. H. Hu, L. X. Shi and W. Min, Nat. Methods 16 (9), 830-842 (2019).

47. L. X. Shi, F. H. Hu and W. Min, Nature Communications 10 (2019).

48. D. Fu, G. Holtom, C. Freudiger, X. Zhang and X. S. Xie, J. Phys. Chem. B 117 (16), 4634-4640 (2013).

49. T. Hellerer, A. M. K. Enejder and A. Zumbusch, Appl. Phys. Lett. 85 (1), 25-27 (2004).

50. I. Pope, W. Langbein, P. Borri and P. Watson, Method Enzymol 504, 273-291 (2012).

51. L. Wei, Z. X. Chen, L. X. Shi, R. Long, A. V. Anzalone, L. Y. Zhang, F. H. Hu, R. Yuste, V. W. Cornish and W. Min, Nature 544 (7651), 465-+ (2017).

52. D. Fu, T. Ye, T. E. Matthews, G. Yurtsever and W. S. Warren, BIOMEDO 12 (5) (2007).

53. D. L. Zhang, M. N. Slipchenko, D. E. Leaird, A. M. Weiner and J. X. Cheng, Opt. Express 21 (11), 13864-13874 (2013).

54. N. Sperelakis, Heart physiology and pathophysiology, 4th ed. (Academic Press, San Diego, 2001).

55. K. Sekiguchi, S. Yamaguchi and T. Tahara, J. Phys. Chem. A 110 (8), 2601-2606 (2006).

56. D. Laage, T. Elsaesser and J. T. Hynes, Chem. Rev. 117 (16), 10694-10725 (2017).

57. R. Milo, Bioessays 35 (12), 1050-1055 (2013).

58. M. Feric, N. Vaidya, T. S. Harmon, D. M. Mitrea, L. Zhu, T. M. Richardson, R. W. Kriwacki, R. V. Pappu and C. P. Brangwynne, Cell 165 (7), 1686-1697 (2016).

59. C. S. Liao, K. C. Huang, W. L. Hong, A. J. Chen, C. Karanja, P. Wang, G. Eakins and J. X. Cheng, Optica 3 (12), 1377-1380 (2016). 